\title{
Promoting a healthy gut microbiome in preterm infants
}

\section{Natalie V. Scime ${ }^{1}$}

1. Western Univeristy

\section{Introduction}

Establishing a healthy gut microbiome early in life is deemed essential for lifelong health, and recent research has begun to explore how profoundly important this is for preterm infants (1). Gut microbiome growth occurs in utero, during, and after birth, and is influenced by several factors such as mode of delivery, gestational age, type of feeding, environment of care, and antibiotic use $(2,3)$. In healthy newborns, gut microbiome development begins with facultative anaerobes such as Lactobacillus, followed by the colonization of anaerobic genera including Bifidobacterium and Clostridium (2). Preterm infants demonstrate distinct imbalances of these microbiota, termed dysbiosis, putting them at risk for several adverse health outcomes (2). Specifically, the gut microbiome of preterm infants shows markedly decreased bacterial diversity, a lack of Bifidobacterium and Lactobacillus, and an increased colonization of potentially pathogenic microorganisms called Proteobacteria $(2,4)$. Preterm infants born via caesarean section are at an even further disadvantage than those born vaginally, as the lack of maternal vaginal and epithelial flora exposure results in further abnormal alterations during gut microbiome development (5).

\section{Consequences of Dysbiosis}

Dysbiosis largely impacts the immune system, as the immune system and gut microbiome interact to establish normal digestive capabilities, build immune tolerance to foods and select antigens, and protect against pathogens (6). Thus, the low diversity of gut microbiota and resulting dysfunctional immune system are associated with a compromised gastric mucosal integrity. This perturbation and ensuing biochemical cascade may contribute to the development of life-threatening infections including sepsis and necrotizing enterocolitis (NEC) $(1,4,6)$. Researchers have used stool sample analysis to confirm this, finding that preterm infants who develop NEC demonstrate significantly less bacterial diversity in their gut microbiome than controls (3). In addition to these imminent risks, asthma, obesity, diabetes, autism, depression, and inflammatory bowel disease are among the many long-term health conditions associated with abnormal gut microbial colonization in early life $(2,5,7)$. Evidently, resolving this dysbiosis to promote healthy gut bacteria is essential for the survival and health of these vulnerable infants.

\section{Probiotics}

Probiotic use has been under study in the preterm neonatal population to rectify such microbial imbalances. Favourable outcomes have been observed in select clinical trials, including an increase in gut colonization and reduction in the incidence of NEC following probiotic administration $(6,8)$. However, some concerns about the quality and scope of probiotic research to date as well as the regulation of these products seem to persist. Probiotics must survive in the gastrointestinal tract at high levels, however the minimum effective dose at which this occurs has yet to be elucidated (6). Reviews examining the safety and efficacy of probiotic use have concluded that there is a lack of systematic adverse events reporting in probiotic intervention studies coupled with poor intervention documentation (6). There is a need for the use of head-to-head studies in addition to improved and standardized detail reporting (i.e., type, duration, and amount of probiotic) to strengthen this knowledge base further (6). Outside of controlled research, probiotic supplementation has not been widely adopted in North American neonatal intensive care units (NICUs). This is partially due to concerns about the lack of a rigorous regulatory framework for these products, as well as difficulties associated with developing a domestically available formulation with evidence-based efficacy and safety (4). Probiotic administration to preterm infants likely requires additional research and practitioner uptake before 
it will be fully integrated into practice.

\section{Breastfeeding and Skin-to-Skin Contact}

While the research and adoption of probiotic use is still in progress, lower risk avenues have been identified as promising interventions to develop the gut microbiome. Skin-to-skin contact is becoming widely encouraged in NICUs and consists of placing the infant between their mother's breasts on her bare chest (9). This practice has numerous health benefits including the promotion of microbial transfer and maintenance of lactogenesis (i.e., the production of breast milk) (5). Although minimal studies have directly examined skin-to-skin contact and microbiome development (9), researchers consistently cite this practice as having a positive influence on gut microbiome acquisition and diversity $(2,5,10)$. Breastfeeding already has an extensive portfolio of health advantages, and is now regarded as an important mechanism for promoting gut microbiome health (4). Breast milk contains several bioactive compounds, such as immunoglobulins, cytokines, and oligosaccharides, which orchestrate the development of gut microbial communities (3). For example, feeding infants with breast milk in the NICU has significantly reduced the incidence of sepsis and NEC, and this reduction has been linked in part to the beneficial microbial species introduced into the infant gut by breast milk (1).

\section{Conclusion}

While evidence continues to accumulate, it can be concluded that addressing the microbiome in preterm infants and encouraging its diversity should be a priority for healthcare providers and researchers. Probiotics have demonstrated effectiveness in various clinical trials and are presently endorsed for use among preterm infants by the Canadian Pediatric Society (4). However, continued studies with improved designs and documentation will help to inform practice guidelines and persuade providers. More research is needed to solidify the benefits of skin-to-skin contact and breastfeeding in promoting healthful gut microbiome development, specifically in preterm infants. Nonetheless, these two practices represent low-cost and low-risk means to develop the preterm infant gut microbiome and can be championed by NICU staff for optimizing the acute and long-term health of these infants.

\section{References}

1. Groer MW, Luciano AA, Dishaw LJ, Ashmeade TL, Miller E, Gilbert JA. Development of the preterm infant gut microbiome: A research priority. Microbiome. 2014;2(38).

2. Cong $X$, Henderson WA, Graf J, McGrath JM. Early life experience and gut microbiome. Adv Neonatal Care. 2015;15(5):314-23.

3. Neu J. The microbiome and its impact on disease in the preterm patient. Curr Pediatr Rep. 2013;1(4):215-21.

4. Unger S, Stintzi A, Shah P, Mack D, O'Connor DL. Gut microbiota of the verylow-birth-weight infant. Pediatr Res. 2015;77(1-2):205-13.

5. Groer MW, Gregory KE, Louis-Jacques A, Thibeau S, Walker WA. The very low birth weight infant microbiome and childhood health. Birth Defects Res Part C Embryo Today Rev. 2015;105:252-64.

6. Gritz EC, Bhandari V. The human neonatal gut microbiome: A brief review. Front Pediatr. 2015;3:17.

7. Gregory KE. Microbiome aspects of perinatal and neonatal health. J Perinat Neonatal Nurs. 2011;25(2):158-62.

8. Abdulkadir B, Nelson A, Skeath T, Marrs ECL, Perry JD, Cummings SP, et al. Routine Use of Probiotics in Preterm Infants: Longitudinal Impact on the Microbiome and Metabolome. Neonatology. 2016 Feb 10;109(4):239-47.

9. Hartz LE, Bradshaw W, Brandon DH. Potential NICU environmental influences on the neonate's microbiome: A systematic review. Adv Neonatal Care. 2015;15(5):324-35.

10. Gregory KE, LaPlante RD, Shan G, Kumar DV, Gregas M. Mode of birth influences preterm infant intestinal colonization with bacteroides over the

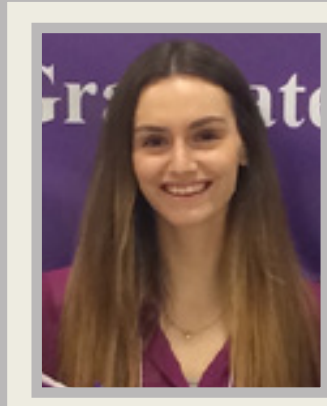

\section{Natalie V. Scime}

Natalie recently graduated with distinction from a Bachelor of Health Sciences at Western University and is now pursuing a Master's degree in Health Promotion, also at Western. She is the recipient of a Canadian Institutes of Health Research Frederick Banting and Charles Best Canada Graduate Scholarship Master's Award for 2015-2016. Her thesis focuses on breastfeeding support for mothers of preterm infants in neonatal intensive care units across Canada. Natalie's research interests include maternal, infant, and child health, as well as knowledge translation. 